\title{
The New York pilot newborn screening program for lysosomal storage diseases: Report of the First 65,000 Infants
}

\author{
Melissa P. Wasserstein, MD ${ }^{1}$, Michele Caggana, Sc.D. ${ }^{2}$, Sean M. Bailey, MD, \\ Robert J. Desnick, PhD, $\mathrm{MD}^{4}$, Lisa Edelmann, $\mathrm{PhD}^{4}$, Lissette Estrella, $\mathrm{NP}^{4}$, Ian Holzman, $\mathrm{MD}^{4}$, \\ Nicole R. Kelly, $\mathrm{MPH}^{1}$, Ruth Kornreich, $\mathrm{PhD}^{4}$, S. Gabriel Kupchik, MD ${ }^{5}$, Monica Martin, PhD², \\ Suhas M. Nafday, MD ${ }^{1}$, Randi Wasserman, $\mathrm{MD}^{6}$, Amy Yang, $\mathrm{MD}^{4}$, Chunli $\mathrm{Yu}, \mathrm{MD}^{4}$ and \\ Joseph J. Orsini, $\mathrm{PhD}^{2}$
}

\begin{abstract}
Purpose: We conducted a consented pilot newborn screening (NBS) for Pompe, Gaucher, Niemann-Pick A/B, Fabry, and MPS 1 to assess the suitability of these lysosomal storage disorders (LSDs) for public health mandated screening.

Methods: At five participating high-birth rate, ethnically diverse New York City hospitals, recruiters discussed the study with postpartum parents and documented verbal consent. Screening on consented samples was performed using multiplexed tandem mass spectrometry. Screen-positive infants underwent confirmatory enzymology, DNA testing, and biomarker quantitation when available. Affected infants are being followed for clinical management and long-term outcome.
\end{abstract}

Results: Over 4 years, 65,605 infants participated, representing an overall consent rate of $73 \%$. Sixty-nine infants were screenpositive. Twenty-three were confirmed true positives, all of whom were predicted to have late-onset phenotypes. Six of the 69 currently have undetermined disease status.

Conclusion: Our results suggest that NBS for LSDs is much more likely to detect individuals at risk for late-onset disease, similar to results from other NBS programs. This work has demonstrated the feasibility of using a novel consented pilot NBS study design that can be modified to include other disorders under consideration for public health implementation as a means to gather critical evidence for evidence-based NBS practices.

Genetics in Medicine (2019) 21:631-640; https://doi.org/10.1038/s41436018-0129-y

Key words: newborn screening; pilot newborn screen; lysosomal storage disorders; informed consent

\section{INTRODUCTION}

The lysosomal storage disorders (LSDs) comprise a group of more than 50 multisystem diseases with a combined frequency of less than 1:7000 (ref. ${ }^{1}$ ). Depending on their specific genotypes, patients can present with severe early infantile or milder late-onset phenotypes. For some, the lateonset phenotypes are significantly more prevalent than the early-onset forms. Many LSDs are treatable with various therapies including enzyme replacement, pharmaceutical chaperones, substrate reduction therapy, and/or bone marrow transplantation. ${ }^{2}$ Because early treatment is assumed to optimize outcomes, there is interest in screening newborns for LSDs with the goal of reducing disease-related morbidity and mortality through early detection.
Newborn screening (NBS) for LSDs began more than ten years ago, when $\sim 37,000$ Italian newborns were screened for Fabry disease. ${ }^{3}$ Subsequently, Taiwan initiated screening for Pompe disease, ${ }^{4}$ followed by Fabry disease a few years later. ${ }^{5}$ These programs demonstrated the feasibility of screening and provided evidence about the disease incidence in these populations. In addition, screening identified newborns with infantile Pompe disease who benefited from early treatment. ${ }^{6}$ Anonymous retrospective pilot studies in Washington, Italy, and Hungary provided statistics on expected incidence rates for various LSDs. ${ }^{3,7-10}$ All of these programs screen dried blood spot (DBS) samples for reduced lysosomal enzyme activities. Some states include DNA and/or biochemical testing as second-tier tests, yet despite this, with rare

\footnotetext{
${ }^{1}$ Albert Einstein College of Medicine and the Children's Hospital at Montefiore, Bronx, New York, USA; ${ }^{2}$ Division of Genetics, Wadsworth Center, New York State Department of Health, Albany, New York, USA; ${ }^{3}$ New York University School of Medicine and NYU Langone Health, New York, New York, USA; ${ }^{4}$ Icahn School of Medicine at Mount Sinai and the Mount Sinai Medical Center, New York, New York, USA; ${ }^{5}$ Maimonides Medical Center, Brooklyn, New York, USA; ${ }^{6}$ New York City Health and Hospitals/Elmhurst, Queens, New York, USA. Correspondence: Melissa P. Wasserstein (mwassers@montefiore.org)
} 
exception (e.g., detection of the $30-\mathrm{KB}$ deletion or very elevated psychosine in Krabbe disease) these NBS assays cannot distinguish between severe early-onset and milder late-onset phenotypes.

Whether newborns should be screened for LSDs is a complex issue. At first glance, these diseases are ideally suited for NBS, as their markers are readily detectable on DBS and there are approved treatments. However, the reality of screening newborns for LSDs raises unique issues that are primarily related to the inevitable detection of newborns with late-onset phenotypes, and the uncertain benefits and unknown risks of doing so. Clearly, important information needs to be collected about the LSDs to facilitate decisionmaking about their suitability for public health mandated screening.

Pilot NBS studies are performed when there are questions about the feasibility, accuracy, and efficacy of newborn screening for certain disorders, such as LSDs. ${ }^{11}$ In contrast to routine NBS, pilot NBS are research studies designed to answer specific questions. The importance of pilot studies is underscored in Public Law 113-240 Sec 116 of the Newborn Screening Saves Lives Reauthorization Act of 2014, which encourages the National Institutes of Health (NIH) to "conduct pilot studies on conditions recommended by the Advisory Committee to ensure that screenings are ready for nationwide implementation." Most pilot NBS are anonymous, meaning that de-identified samples are tested to measure screening feasibility and disease incidence. ${ }^{3,7-10}$ Because this approach eliminates the connection between the sample and the patient, results from anonymous pilot NBS studies are not reported to families, clinical diagnosis and outcome data is not collected, and there is no way to confirm the positive predictive value of the assay. Identifiable pilot studies that maintain the link between the NBS dried blood spot (DBS) and the infant, such as those performed in Massachusetts ${ }^{12-14}$ and California, ${ }^{15}$ are an ideal way to gather this information. However, they are challenging to implement as they require efficient ways to educate and consent thousands of parents, sort consented versus nonconsented samples, track and crosscheck NBS results, and ensure appropriate results reporting and clinical follow up. ${ }^{11}$

New York's pilot LSD NBS program has successfully worked through these consenting and infrastructure challenges. We now report the screening results of the 65,605 infants who participated in the study. Because it is an ongoing study, outcome data continue to be collected, as well as valuable information about the unique ethical, legal, and social issues (ELSI) associated with NBS for these disorders. The laboratory screening data, pilot screen infrastructure, and ELSI data will be described in separate reports.

\section{Study subjects}

\section{MATERIALS AND METHODS}

Study recruitment was initiated in May 2013. Six NYC hospitals have participated in the pilot screen: Mount Sinai Medical Center and NYU Langone Health in Manhattan;
Elmhurst Hospital Center in Queens; Maimonides Medical Center in Brooklyn; and the Jack D. Weiler Hospital of the Albert Einstein College of Medicine for recruitment, and the Children's Hospital at Montefiore in the Bronx for coordination and patient follow up. The study was approved by the institutional review boards (IRBs) from each institution and the New York State Department of Health. All infants born at the pilot hospitals were eligible to participate regardless of gender, gestational age, or health status. Parental consent to participate was obtained within the first $48 \mathrm{~h}$ after birth. Study recruiters approached parents to discuss the pilot screen, provide study and disease information using multilingual brochures and videos, and document verbal consent. On weekends and other times when the recruiters were not present, parents had the option to enroll by filling out brochures and placing them in locked drop boxes on the postpartum wards, which were then collected by the recruiters. The NBS cards from consented specimens were distinctly marked with a study-specific stamp. Consented specimens were sent to the New York State NBS Lab in a clearly marked "study envelope," separately from nonconsented specimens, which were sent per the hospital's usual practice. Consented, marked specimens had routine NBS testing performed first, and were then transferred to the New York NBS LSD laboratory (located in the same building) for the additional testing. All LSD testing was performed using DBS specimens already collected for routine NBS; no additional blood was collected for the pilot study.

\section{Screening assay}

All consented specimens were screened for Fabry, Gaucher, and Niemann-Pick A/B (also called Acid Sphingomyelinase Deficiency, or ASMD). Initially, Pompe disease was included in the pilot screen panel, but it was removed on 1 October 2014 at which time it was added to New York's routine NBS panel. In May 2015, mucopolysaccharidosis type 1 (MPS 1) was added to the pilot screen panel.

Screening was performed on DBS using the LSD $4+1$ or LSD 6-plex tandem mass spectrometry methods as previously described. ${ }^{9,16}$ Substrate and internal standard pairs were provided by the Centers for Disease Control and Prevention (CDC " $4+1$ " assay) until May 2015, and PerkinElmer (PE "6-plex" assay) thereafter. ${ }^{17}$ While mean activity for some analytes changed as a result of the reagent change from CDC to PerkinElmer, our use of percent daily mean cutoffs allowed us to keep the algorithm constant despite the assay switch.

Based on experience during method optimization, early specimens were retested in duplicate if the enzyme activity was less than $20 \%$ of the population mean, as the number of batched samples was small during the first few months of the study. Later, as sample numbers increased, specimens were retested if the enzyme activity was less than $20 \%$ of the daily mean activity (DMA). For those samples that were retested, if the average of the three replicates was below the referral cutoff of $15 \%$ (Pompe, Gaucher, Niemann-Pick A/B, and MPS 1), the specimen was considered screen-positive. The cutoff of 
Table 1 Diagnostic determinations based on confirmatory testing results

\begin{tabular}{|c|c|c|c|}
\hline & Enzyme activity & Variant(s) & Biomarkers \\
\hline True positive & Within laboratory's affected range & Known variants or likely pathogenic alleles & $\begin{array}{l}\text { Elevated GAGs (MPS 1) } \\
\text { or Glc4 (Pompe) }\end{array}$ \\
\hline Not affected & $\begin{array}{l}\text { Low, but above laboratory's } \\
\text { affected range, or within } \\
\text { laboratory's normal range }\end{array}$ & $\begin{array}{l}\text { No variants, or carrier (one variant plus wild type or } \\
\text { pseudodeficiency allele) or two pseudodeficiency alleles }\end{array}$ & $\begin{array}{l}\text { Normal GAGs (MPS 1) } \\
\text { or Glc4 (Pompe) }\end{array}$ \\
\hline $\begin{array}{l}\text { Undetermined } \\
\text { disease status }\end{array}$ & $\begin{array}{l}\text { Low, but above laboratory's } \\
\text { affected range }\end{array}$ & $\begin{array}{l}\text { Two variants of uncertain significance, or one variant } \\
\text { of uncertain significance and one known or likely } \\
\text { pathogenic allele }\end{array}$ & $\begin{array}{l}\text { Normal GAGs (MPS 1) } \\
\text { or GIc4 (Pompe) }\end{array}$ \\
\hline
\end{tabular}

"True positive" denotes all disease phenotypes, regardless of predicted severity or age of onset.

GAG, glycosaminoglycan; MPS 1, mucopolysaccharidosis type 1

Table 2 Overall screening results

\begin{tabular}{|c|c|c|c|c|c|c|c|}
\hline & & Pompe & Gaucher & ASMD & Fabry & MPS 1 & Totals \\
\hline \multicolumn{2}{|l|}{ Screen positives (rate) } & $6(0.033 \%)$ & $17(0.026 \%)$ & $2(0.003 \%)$ & $31(0.047 \%)$ & $13(0.036 \%)$ & $69(0.11 \%)$ \\
\hline \multicolumn{2}{|l|}{ True positive } & 1 & 15 & 0 & 7 & 0 & 23 \\
\hline \multirow[t]{2}{*}{ Predicted phenotype } & Early onset & 0 & 0 & 0 & 0 & 0 & 0 \\
\hline & Late onset & 1 & 15 & 0 & 7 & 0 & 23 \\
\hline \multicolumn{2}{|l|}{ Heterozygote/carrier } & 2 & 0 & 0 & 2 & 4 & 8 \\
\hline \multicolumn{2}{|l|}{ Not affected } & 3 & 2 & 0 & 18 & 8 & 31 \\
\hline \multirow[t]{2}{*}{ Reason for not affected } & Normal confirmatory results & 0 & 2 & 0 & 12 & 0 & 14 \\
\hline & Pseudodeficiency/benign variant & 3 & NA & NA & 6 & 8 & 17 \\
\hline \multicolumn{2}{|c|}{ Undetermined disease status } & 0 & 0 & 2 & 3 & 1 & 6 \\
\hline \multicolumn{2}{|c|}{ Pending or lost to follow up } & 0 & 0 & 0 & 1 & 0 & 1 \\
\hline
\end{tabular}

MPS 1, mucopolysaccharidosis type 1

$15 \%$ was purposely set high in this pilot screen to conservatively determine the cutoff values that would be used in future implementation of statewide screening, despite the expected higher false positive rate. Due to a lower than expected number of screen-positive infants with low a-galactosidase A activity (for Fabry disease) the retest and referral cutoffs for this analyte were adjusted to $30 \%$ and $25 \%$ DMA respectively.

\section{Referrals and diagnostic evaluations}

Infants with results below the screening cutoff were referred to a study investigator (M.P.W. or A.Y.) for confirmatory testing. Confirmation of leukocyte enzyme activities was performed using synthetic substrates and internal standards from the CDC, with analysis by liquid chromatography-tandem mass spectrometry (LC-MS/ MS). ${ }^{18-21}$ Plasma a-galactosidase A activity was measured using a 4-methylumbelliferyl (4-MU)-a-galactopyranoside assay. $^{22}$ In addition, molecular analysis of the relevant gene was performed. Urine biomarkers were measured in some infants referred for Pompe disease (Glc4 concentration, measured at Duke University) and MPS 1 (glycosaminoglycan, or GAG, quantitation, measured at commercial laboratories).

Clinical diagnoses and phenotypic predictions were based on results of confirmatory testing (Table 1), using a combination of enzyme activity and genotype information for all five disorders, plus biomarker quantification for MPS 1 and Pompe disease. Predicted early-onset phenotypes were defined as classic infantile Pompe disease, types 2 or 3 Gaucher disease, Niemann-Pick type A, type 1 Fabry disease, and severe MPS 1. Predicted late-onset phenotypes were defined as late-onset Pompe disease, type 1 Gaucher, Niemann-Pick type B, type 2 Fabry disease, and attenuated MPS 1 . For the purpose of creating diagnostic determinations, cases with enzyme activity within the affected range in the presence of known variants or likely pathogenic variants were considered to be true positives, regardless of predicted age of onset or clinical symptomatology.

\section{RESULTS}

In total, 89,346 families were approached and/or had the opportunity to participate and 65,605 (73\%) consented to participate. This overall percentage included days when recruiters were not present, such as vacations, holidays, and weekends. On the days when the recruiters were present, the consent rate averaged $86 \%$.

Overall referral numbers and results of confirmatory testing are summarized in Table 2. Sixty-nine infants were screenpositive, and of these, 23 , or $33 \%$, were determined to be true positive based on the criteria shown in Table 1 . All true 
positives were determined to be at risk for late-onset disease; all of these infants had genotypes known to be associated with late-onset phenotypes, low enzyme activity, and none had relevant clinical symptoms at the most recent evaluation. Thirty-one of the 69 referred infants, or $45 \%$, were determined to be not affected; of these, 17 (25\% of referrals) were found to have pseudodeficiency or benign alleles, and the remaining 14 (20\% of referrals) were real false positives with normal confirmatory results. Eight infants carried only single variants or single variants of uncertain significance; two of these were female infants at risk for Fabry disease, four were carriers for MPS 1, and two were carriers for Pompe disease. Six of the 69 screenpositive infants currently have undetermined disease status, although it is likely that this number will decrease as more information about the pathogenicity of specific variants becomes available.

In the Pompe disease study, which ran from study commencement until 1 October 2014, 18,105 initial specimens were screened for GAA activity, and 6 were screenpositive with a mean GAA activity $\leq 15 \%$ of the daily mean activity for a referral rate of $0.033 \%$. Confirmatory testing, genotype, diagnostic determination, and predicted phenotype are shown in Table 3. Two infants were homozygous for the common pseudodeficiency allele, and another carried one copy of the pseudodeficiency allele; confirmatory leukocyte GAA activity in these infants ranged from low to normal (11.6-82.4 nmol/hr/mg protein). One infant was homozygous for the late-onset c.-32-13T>G pathogenic variant $(\mathrm{PV})$ and had low confirmatory GAA activity, normal exam, normal lab values, and negative Glc4 testing. Another infant was found to have a novel VUS (c.2560C $>\mathrm{T}$ ) and an intronic variant believed to be benign based on in silico and functional predictions. This child's GAA activity was $25.6 \mathrm{nmol} / \mathrm{hr} / \mathrm{mg}$ protein, which was well above the laboratory's affected range of $<3.1 \mathrm{nmol} /$ $\mathrm{hr} / \mathrm{mg}$ protein, and the child was diagnosed as being a carrier. The last Pompe screen-positive infant carried one pseudodeficiency allele and a single copy of the late-onset c.-32-13T $>$ G PV, had near normal GAA activity, and was diagnosed as being a carrier.

Of 65,605 DBS tested for Gaucher disease, there were 17 screen positives for a referral rate of $0.026 \%$. Two infants had normal acid $\beta$-glucosidase activity on confirmatory testing, but all others were true positives, with low enzyme activity in the affected range and two known . Of these, all but one were homozygous for the common c.1226A $>$ G (p.N409S) $\mathrm{PV}$, which is associated with type 1, late-onset Gaucher disease (Table 3).

Niemann-Pick A/B screening $(N=65,605)$ resulted in two infants referred for confirmatory testing $(0.002 \%)$. Both of these infants were homozygous for different but previously undescribed variants of uncertain significance. One had low ASM activity that was above the laboratory's affected range, and another had low enzyme activity at the high end of the laboratory's affected range (Table 3 ). At last visit, both were
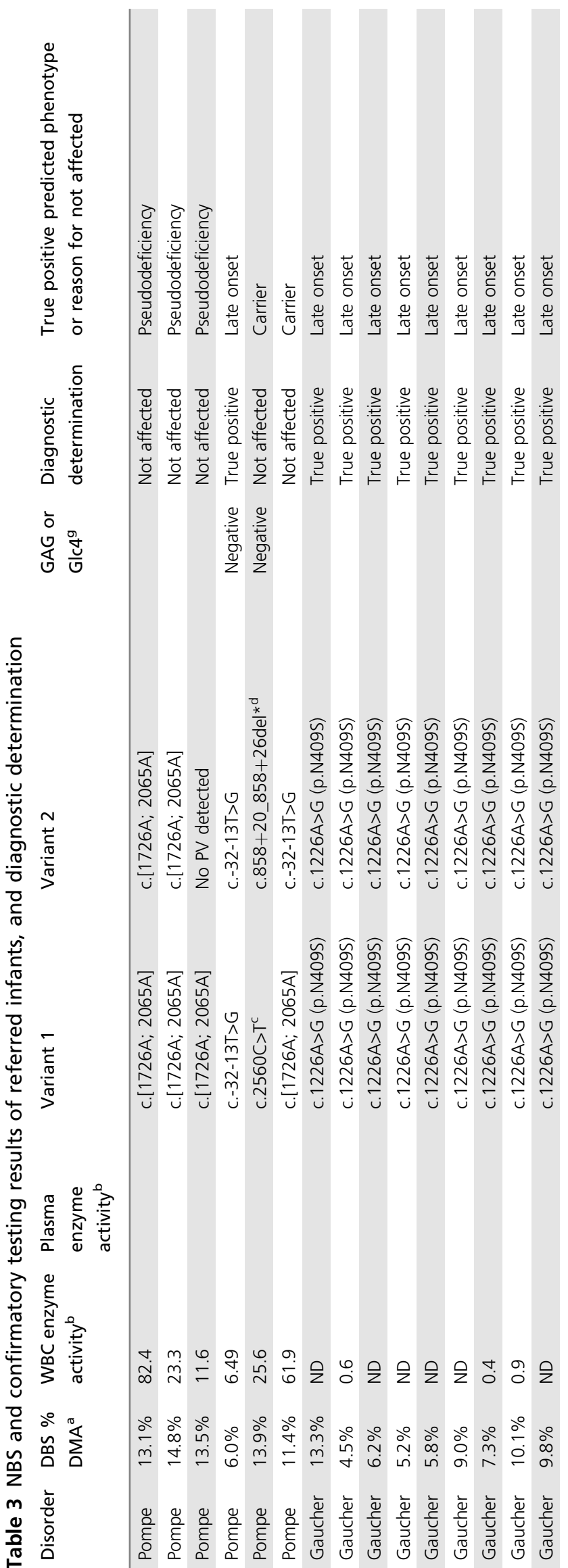


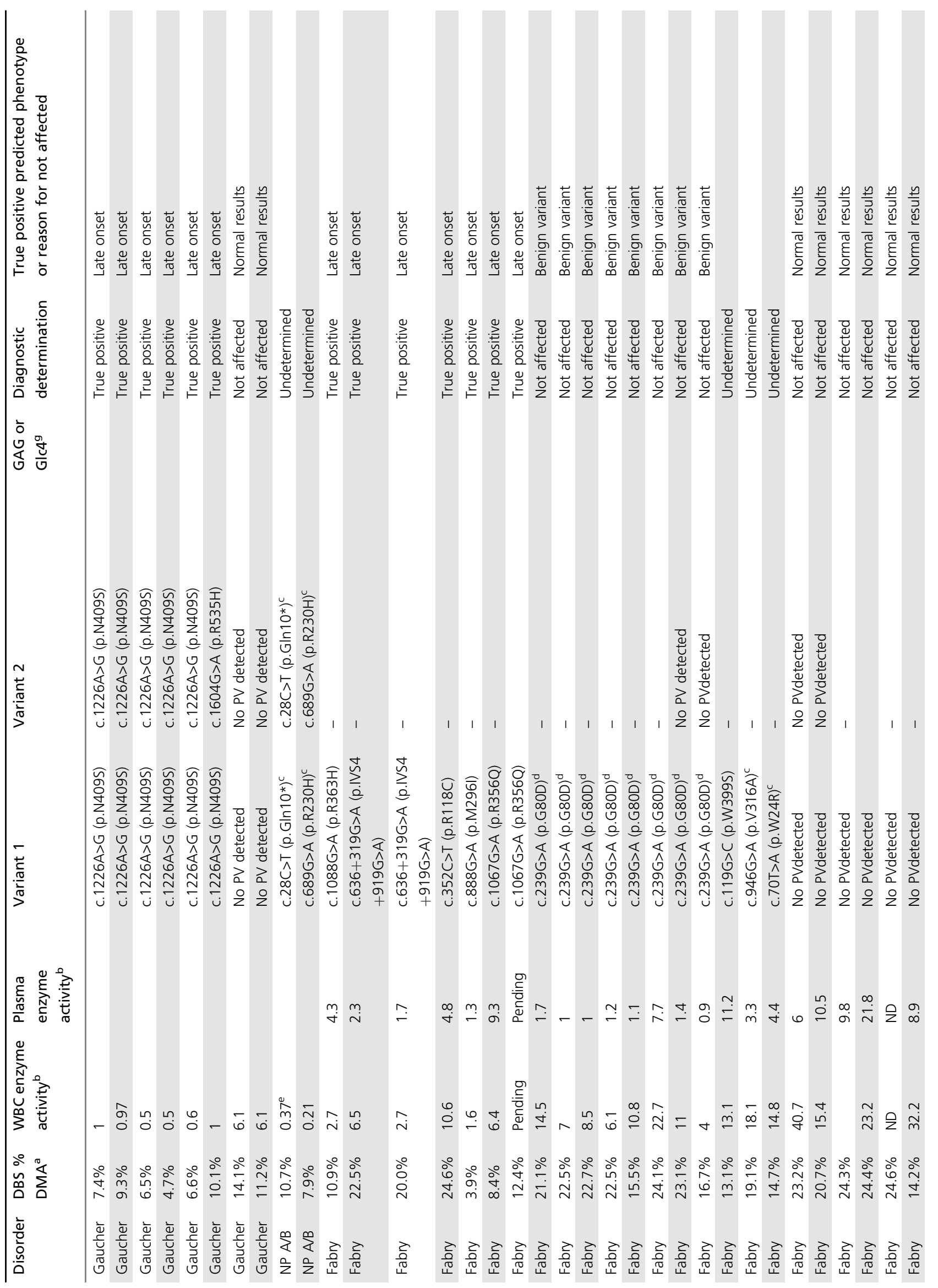




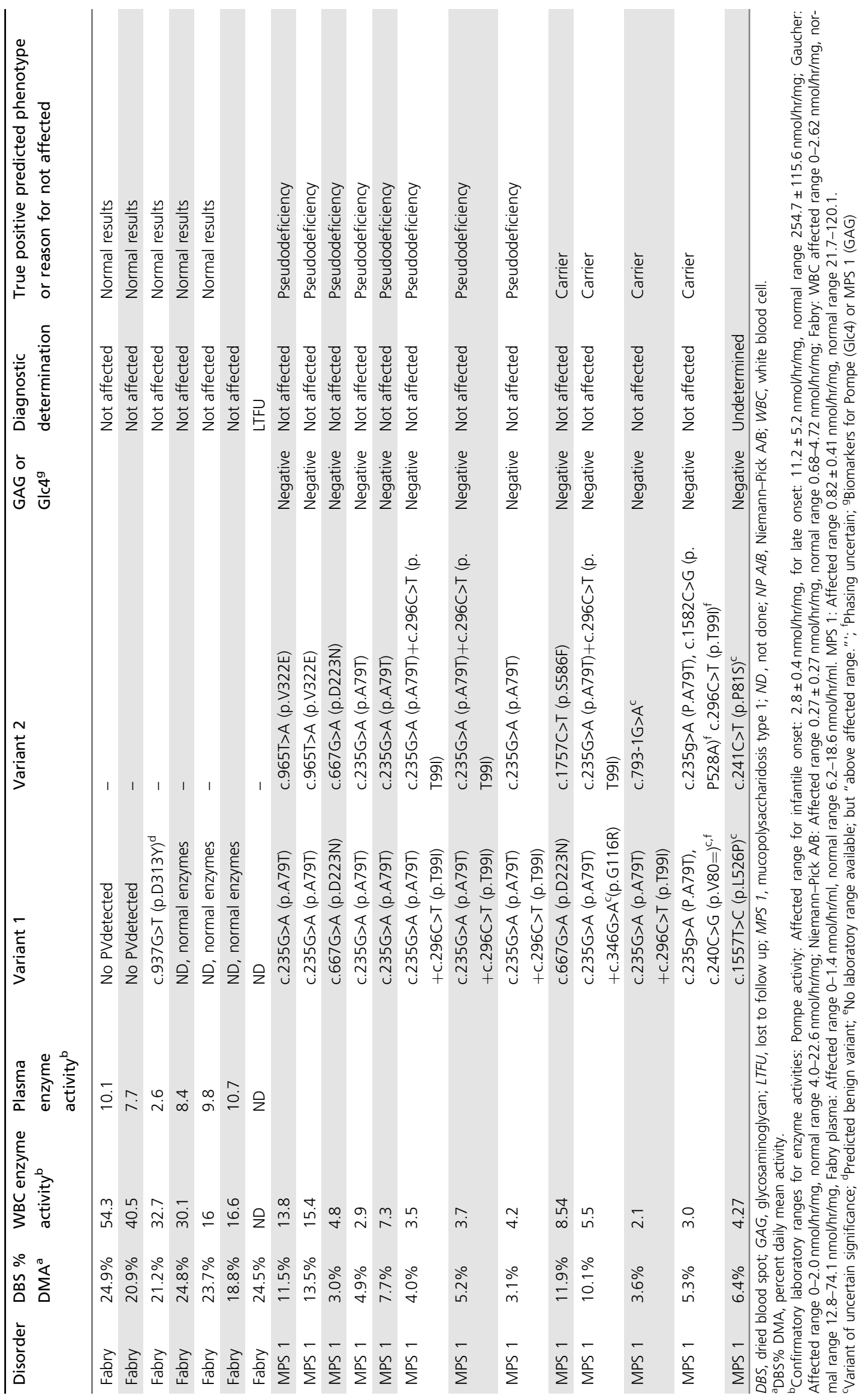


asymptomatic, and both are considered to have undetermined disease status.

In the Fabry study, 65,605 initial specimens were screened resulting in 31 referrals for a screen-positive rate of $0.047 \%$. (Table 2) There were seven infants with low enzyme activity and known PV associated with type 2, late-onset Fabry disease; two of these had the common c.636+319G $>A$ (p.IVS4+919G $>$ A) PV that causes the late-onset cardiac phenotype $^{23}$ (Table 3). Twelve male infants had normal plasma and leukocyte enzyme activity and/or no PV and were considered to be not affected with Fabry disease. Interestingly, eight apparently unrelated Ecuadorian Hispanic infants, including two female heterozygotes, carried the c.239G $>A$ (p.G80D) variant. None of these infants had family members who were diagnosed with or had obvious symptoms of Fabry disease. All of the hemizygous males with p.G80D had leukocyte GLA activity (range $6.1-22.7 \mathrm{nmol} / \mathrm{hr} / \mathrm{mg}$ ) that was well above the affected range $(0-2.62 \mathrm{nmol} / \mathrm{hr} / \mathrm{mg})$. The mean leukocyte GLA activity $(9.65 \mathrm{nmol} / \mathrm{hr} / \mathrm{mg})$, was $28 \%$ of the mean normal GLA activity $(34.6 \mathrm{nmol} / \mathrm{hr} / \mathrm{mg}$, range 12-99.1), which is consistent with recent in vitro expression data showing significant residual enzyme activity. ${ }^{24} 1000$ Genomes reports a relatively high frequency of this variant, 1:143 in the Peruvian population and 1:714 in the admixed American population, supporting its benign nature. ${ }^{25}$ Although the families were initially counseled that the pathogenicity of this variant was uncertain, it now seems likely that this is a benign variant and these children were classified as not affected.

Since testing for IDUA activity commenced in May 2015, 35,816 infants have been tested for MPS 1. Thirteen referrals were made for a rate of $0.036 \%$. As shown in Table 3, all referred infants were found to have leukocyte enzyme activity that was low, but above the laboratory's affected range. Eight infants of the 13 had only pseudodeficiency alleles. Two infants had one pseudodeficiency allele and a likely pathogenic variant, and two infants had two or more pseudodeficiency alleles and a single variant of uncertain significance; all of these infants were considered to be carriers of MPS 1 . Another had two variants of uncertain significance, c.1557T $>C$ (p.L526P) and c.241C >T (p.P81S), in trans, and is considered to have undetermined disease status. Urine glycosaminoglycans (GAGs), which were collected at the initial confirmatory visit within the first few weeks of life, were normal in all infants. (Table 3).

\section{DISCUSSION}

There were 65,605 families who participated in this unique pilot NBS for Pompe, Gaucher, Niemann-Pick A/B, Fabry, and MPS 1 disease. Pilot newborn screens are the ideal way to acquire evidence to support whether a disorder is appropriate for NBS. Because pilot NBS are research studies, data are collected in a meticulous and uniform manner, which permits calculation of disease incidence, spectrum of clinical phenotype detected by screening, optimization of NBS methodology, and determination of
NBS cutoffs and screening algorithms prior to widespread screening. Another major benefit of pilot NBS is the use of informed consent. In fact, it has been suggested that NBS should always involve parental informed consent, ${ }^{26}$ but this is generally considered to be logistically impractical due to several factors, such as the expense and time for personnel to satisfactorily inform all new parents about all of the disorders on routine NBS. For this reason, most NBS programs do not require parental informed consent for routine screening. However, identifiable pilot screens that are designed as research studies with the goals of informing NBS laboratory protocol and gathering important longterm clinical follow-up data should involve parental informed consent.

Our pilot screen utilized an "opt in" mode of parental consent, and was designed to ensure that parents were educated about the additional screening and were active participants in the decision-making process about whether to have their infant tested. The high consent rates suggested that parents were quite amenable to having their infants screened for LSDs. Other states have conducted consented pilot NBS with varying degrees of participation. In 2002-2003, California used a full informed consent model to pilot MS-MS detectable disorders. Only about half of families were offered testing, and while $90 \%$ of those approached agreed to participate, the overall consent rate was $47 \%{ }^{15}$ In contrast, in Massachusetts, there are ongoing, optional pilot NBS where hospital staff discusses the study with parents, and asks them whether they would like to participate. Their consent rate has been as high as $>99 \%{ }^{27}$

In our study, after confirmatory testing, 23 infants were diagnosed with disease, all with phenotypes that typically manifest during adulthood: type 2 late-onset Fabry disease, type 1 Gaucher disease, and late-onset Pompe disease. It is important to note that the inconsistent use of vocabulary to describe age of onset of LSDs (e.g., "juvenile" versus "intermediate" versus "later onset," "late onset" versus "later onset" versus "adult onset") will inevitably complicate how NBS outcome is defined. It will take years, perhaps even decades, to determine if some of these individuals express the disease phenotype. Because of this, our definition of "true positive" includes all phenotypes, regardless of severity and predicted age of onset, and is based on genotypic and biochemical definitions of disease, rather than a definition based on clinical manifestations. While our assumptions may prove incorrect in the future, it is, arguably, the most practical way to discuss NBS results for disorders with variable ages of presentation. The limits of using the term "true positive" in the context of NBS for lateonset phenotypes obligates long-term follow up to determine whether these patients become symptomatic or not; these future phenotypic descriptions are imperative in helping the NBS laboratories determine cutoff and screening algorithms.

One major purpose of pilot NBS is to determine population-specific disease incidence. Importantly, disease 
incidence is only an estimate when screening for late-onset disease, as we assume that all "true positive" infants will develop symptoms. In addition, the total number of infants screened in this study was relatively low. That said, comparisons with other studies can be made. We detected only one late-onset Pompe case $(1: 19,197)$, somewhat lower than that found in Missouri's reported NBS experience $(1: 14,567)\left(\right.$ ref. $\left.^{28}\right)$ but similar to Illinois' observed incidence of 1:21,979 (ref. $^{29}$ ). Our incidence for Gaucher disease was significantly higher $(1: 4374)$ than in other pilot NBS, where it was approximately 1:43,000 (refs. ${ }^{28,29}$ ) All of our infants confirmed to have type 1 Gaucher disease were of Ashkenazi Jewish descent, and our higher incidence likely reflects New York City's large Jewish population. For type 2 lateonset Fabry disease, our observed incidence rate was 1:9372, which was lower than the previously reported incidence rates in Japan (1:7057) (ref. $\left.^{30}\right)$, Italy (1:1100) (ref. $\left.{ }^{3}\right)$, Taiwan (1:1250) (ref. ${ }^{31}$ ), Illinois (1:8454 with p.A143T, or $1: 21,973$ excluding p.A143T) ${ }^{29}$ and Missouri $(1: 2913)\left(\right.$ ref. $\left.^{28}\right)$. We did not detect any affected individuals with MPS 1 or Niemann-Pick A/B; because the incidence of these disorders is estimated to be less than 1 in 100,000 $\left(\mathrm{ref}^{1}\right)$, these findings were not unexpected.

Our observed disease incidences presumably reflect population differences; our pilot screen was performed in New York City because of its genetic diversity, which likely differs significantly from the populations in other pilot screens. It is likely that statewide screening would have a lower incidence of Gaucher disease. It may also reflect differences in our screening assays. The MS/MS $4+1$ multiplex method that we used has also been used in LSD pilot studies in Washington ${ }^{17}$ and Taiwan. ${ }^{5}$ In contrast, Missouri uses a digital microfluidics platform to screen newborns for Pompe, Fabry, Gaucher, and Krabbe disease as part of its routine NBS. ${ }^{28}$ Other NBS programs have used fluorometric based assays. ${ }^{7,30}$ Because of the difficulties with defining true positives for late-onset LSDs, it is difficult to compare and contrast the accuracy of different pilot screens' methodologies and determination of disease incidences. This is further compounded by the issue that NBS laboratories may use different referral cutoffs, even when using the same technologies.

Fabry disease has been included in several pilot NBS studies ${ }^{9,30,31}$ and is now on some routine NBS panels in the United States. Interestingly, we found no infants with the p. A143T Fabry variant, although it seems to be a fairly common variant. $^{10,29,32,33}$ In contrast, $26 \%$ of babies who were screenpositive for Fabry disease in our study carried the p.G80D (c.239G >A) variant; all of these infants were of Ecuadorian descent. Because this variant is associated with significant residual enzyme activity ${ }^{24}$ and has a fairly high frequency in the admixed American population, ${ }^{25}$ we interpret it as likely benign.

Not unexpectedly, $100 \%$ of our pilot screen infants who were confirmed to have disease are at risk for late-onset phenotypes. None of these patients are currently receiving disease-specific treatment. Our findings are similar to LSD screening results in Illinois, where, after screening over 219,000 samples, there were four infants with early-onset LSD phenotypes and 25 at risk for late-onset LSDs. ${ }^{29}$ Both the American Academy of Pediatrics and the American College of Medical Genetics and Genomics (ACMG) recommend against testing children for adult-onset genetic diseases, except in limited circumstances. ${ }^{34,35}$ Thus, there is an obvious clash between these positions and the reality of NBS for LSDs. Data from empirical studies evaluating the risks and benefits of screening newborns for late-onset diseases are needed to provide policy makers with information on which to formulate their guidance on if, and how, they should perform NBS for diseases that include late-onset phenotypes.

With the exception of one infant who moved after a diagnosis of type 2 Fabry disease, and another who was lost to follow up prior to confirmatory testing, most of the children identified through the pilot screen are currently receiving follow-up evaluations with one of the study investigators (M.P.W. or A.Y.). This collection of long-term follow-up data is necessary to assess the impact of NBS on disease outcome, and is the primary focus of LSD registries that were developed in conjunction with the Newborn Screening Translational Research Network (NBSTRN). ${ }^{36}$ Long-term follow up is also required to measure the positive predictive value of NBS for disorders with late-onset phenotypes, to determine whether screen-positive cases are, in fact, true positives, and for adjusting cutoffs or other tools used to determine screen positives. Thus, another benefit of pilot NBS is that they create an avenue for engaging patients in natural history studies and longitudinal patient registries such as these.

Importantly, our results identified issues that should be addressed prior to widespread NBS for Pompe, Gaucher, ASMD, Fabry, and MPS 1 disease. Although this pilot screen used only enzyme assays as a screening method, the use of second-tier DNA testing at the NBS laboratory would likely have resulted in fewer referrals. For example, infants with Pompe and MPS 1 pseudodeficiency alleles were screenpositive and referred for confirmatory testing. Molecular analysis as a second-tier NBS test could have excluded these infants from being referred, reducing the financial and possible psychological costs of unnecessary false positives and confirmatory testing. Accuracy may be improved through the use of second-tier biochemical and molecular testing and data analytic tools such as the Mayo Clinic Collaborative Laboratory Integrated Reports (CLIR) tool, which has been shown to significantly reduce the number of false positives in Kentucky's LSD NBS program. ${ }^{37-40}$

In sum, our pilot screen has demonstrated that the feasibility of conducting a high volume, identifiable consented pilot NBS for LSDs. We found that all of the infants identified through this screen are at risk for late-onset disease, which raises unique ethical and practical issues. Future pilot NBS studies may utilize this infrastructure to obtain important information on other diseases under consideration for widespread NBS implementation. 


\section{ACKNOWLEDGEMENTS}

Special thanks to Dalia Makarem, Aliza Quinones, Katherine Carome, Rebecca Zarchin, Tori Velez, Ryan Wilson, Chad Biski, and Christopher Johnson, and to Priya Kishnani and Deeksha Bali for their expert advice. Research reported in this publication was supported by the Eunice Kennedy Shriver National Institute of Child Health and Human Development of the National Institutes of Health under award number R01HD073292. This research was facilitated by the Newborn Screening Translational Research Network (NBSTRN), which is funded by the Eunice Kennedy Shriver National Institute of Child Health and Human Development, National Institutes of Health (HHSN275201300011C).

\section{DISCLOSURE}

Melissa Wasserstein has served as a consultant for and has received travel reimbursement from Sanofi Genzyme. Robert J. Desnick is a consultant for Amicus Therapeutics, Sanofi Genzyme, and Sangamo Therapeutics. He has received research grants from Sanofi Genzyme and Sangamo Therapeutics. He owns founder's shares for Amicus Therapeutics and options for Sangamo Therapeutics. He has patents for Fabry and Niemann-Pick treatments and receives royalties from Sanofi Genzyme. Lisa Edelmann is an employee of Sema4, a for-profit genetic testing laboratory. Ruth Kornreich is an employee of Sema4, a for-profit genetic testing laboratory. Amy Yang has served as a consultant for Shire Therapeutics. Chunli Yu is an employee of Sema4, a forprofit genetic testing laboratory. The other authors declare no conflicts of interest.

\section{REFERENCES}

1. Wang RY, Bodamer OA, Watson MS, Wilcox WR, ACMG Work Group on Diagnostic Confirmation of Lysosomal Storage Diseases. Lysosomal storage diseases: diagnostic confirmation and management of presymptomatic individuals. Genet Med. 2011;13:457-484.

2. Desnick RJ. Enzyme replacement and enhancement therapies for lysosomal diseases. J Inherit Metab Dis. 2004;27:385-410.

3. Spada M, Pagliardini S, Yasuda M, et al. High incidence of later-onset Fabry disease revealed by newborn screening. Am J Hum Genet. 2006;79:31-40

4. Chien YH, Chiang SC, Zhang XK, et al. Early detection of Pompe disease by newborn screening is feasible: results from the Taiwan screening program. Pediatrics. 2008;122:e39-45.

5. Liao HC, Chiang CC, Niu DM, et al. Detecting multiple lysosomal storage diseases by tandem mass spectrometry - a national newborn screening program in Taiwan. Clin Chim Acta. 2014;431:80-86.

6. Chien $\mathrm{YH}$, Lee NC, Huang $\mathrm{HJ}$, et al. Later-onset Pompe disease: early detection and early treatment initiation enabled by newborn screening. J Pediatr. 2011;158:1023-7e1.

7. Paciotti S, Persichetti E, Pagliardini S, et al. First pilot newborn screening for four lysosomal storage diseases in an Italian region: identification and analysis of a putative causative mutation in the GBA gene. Clin Chim Acta. 2012;413:1827-1831

8. Wittmann J, Karg E, Turi S, et al. Newborn screening for lysosomal storage disorders in Hungary. JIMD Rep. 2012;6:117-125.

9. Elliott S, Buroker N, Cournoyer JJ, et al. Pilot study of newborn screening for six lysosomal storage diseases using tandem mass spectrometry. Mol Genet Metab. 2016;118:304-309.

10. Burlina $A B$, Polo $G$, Salviati $L$, et al. Newborn screening for lysosomal storage disorders by tandem mass spectrometry in North East Italy. J Inherit Metab Dis. 2018;41:209-219.

11. Botkin JR, Lewis MH, Watson MS, et al. Bioethics and Legal Work Group of the Newborn Screening Translational Research Network. Parental permission for pilot newborn screening research: guidelines from the NBSTRN. Pediatrics. 2014;133:e410-7.

12. Zytkovicz TH, Fitzgerald EF, Marsden D, et al. Tandem mass spectrometric analysis for amino, organic, and fatty acid disorders in newborn dried blood spots: a two-year summary from the New England Newborn Screening Program. Clin Chem. 2001;47:1945-1955.

13. Comeau AM, Eaton RB, Successes of newborn screening programs. Science. 2002;295:44-45.

14. Comeau AM, Parad RB, Dorkin HL, et al. Population-based newborn screening for genetic disorders when multiple mutation DNA testing is incorporated: a cystic fibrosis newborn screening model demonstrating increased sensitivity but more carrier detections. Pediatrics. 2004;113:1573-1581.

15. Feuchtbaum L, Lorey F, Faulkner L, et al. California's experience implementing a pilot newborn supplemental screening program using tandem mass spectrometry. Pediatrics. 2006;117(5 pt 2): S261-9.

16. Orsini JJ, Martin MM, Showers AL, et al. Lysosomal storage disorder $4+1$ multiplex assay for newborn screening using tandem mass spectrometry: application to a small-scale population study for five lysosomal storage disorders. Clin Chim Acta. 2012:413:1270-1273.

17. Elliott S, Buroker N, Cournoyer JJ, et al. Dataset and standard operating procedure for newborn screening of six lysosomal storage diseases: by tandem mass spectrometry. Data Brief. 2016;8: 915-924.

18. Lin N, Huang J, Violante $S$, et al. Liquid chromatography-tandem mass spectrometry assay of leukocyte acid alpha-glucosidase for postnewborn screening evaluation of Pompe disease. Clin Chem. 2017;63: 842-851.

19. Zhang XK, Elbin CS, Chuang WL, et al. Multiplex enzyme assay screening of dried blood spots for lysosomal storage disorders by using tandem mass spectrometry. Clin Chem. 2008;54:1725-1728.

20. Li Y, Scott CR, Chamoles NA, et al. Direct multiplex assay of lysosomal enzymes in dried blood spots for newborn screening. Clin Chem. 2004;50:1785-1796.

21. Gelb MH, Turecek F, Scott CR, Chamoles NA, Direct multiplex assay of enzymes in dried blood spots by tandem mass spectrometry for the newborn screening of lysosomal storage disorders. J Inherit Metab Dis. 2006;29:397-404.

22. Desnick RJ, Allen KY, Desnick SJ, et al. Fabry's disease: enzymatic diagnosis of hemizygotes and heterozygotes. Alpha-galactosidase activities in plasma, serum, urine, and leukocytes. I Lab Clin Med. 1973;81:157-171

23. Hsu TR, Hung SC, Chang FP, et al. Later onset Fabry disease, cardiac damage progress in silence: experience with a highly prevalent mutation. J Am Coll Cardiol. 2016;68:2554-2563.

24. Lukas J, Scalia S, Eichler S, et al. Functional and clinical consequences of novel alpha-galactosidase A mutations in Fabry disease. Hum Mutat. 2016;37:43-51.

25. 1000genomes. http://www.1000genomes.org, March 8, 2018.

26. Fost $\mathrm{N}$. Informed consent should be a required element for newborn screening, even for disorders with high benefit-risk ratios. J Law Med Ethics. 2016;44:241-255.

27. Pass K, Green NS, Lorey F, et al. Pilot programs in newborn screening. Ment Retard Dev Disabil Res Rev. 2006;12:293-300.

28. Hopkins PV, Campbell C, Klug T, et al. Lysosomal storage disorder screening implementation: findings from the first six months of full population pilot testing in Missouri. J Pediatr. 2015;166:172-177.

29. Burton BK, Charrow J,Hoganson GE, et al. Newborn screening for lysosomal storage disorders in Illinois: the initial 15-month experience. J Pediatr. 2017:190:130-135.

30. Inoue T, Hattori K, Ihara K, et al. Newborn screening for Fabry disease in Japan: prevalence and genotypes of Fabry disease in a pilot study. J Hum Genet. 2013;58:548-552.

31. Hwu WL, Chien YH, Lee NC, et al. Newborn screening for Fabry disease in Taiwan reveals a high incidence of the later-onset GLA mutation c.936 +919G>A (IVS4+919G>A). Hum Mutat. 2009;30:1397-1405.

32. Lenders $M$, Weidemann $F$, Kurschat $C$, et al. Alpha-Galactosidase $A$ p. A143T, a non-Fabry disease-causing variant. Orphanet J Rare Dis. 2016;11:54

33. Doheny $D$, Srinivasan R, Pagant $S$, et al. Fabry disease: prevalence of affected males and heterozygotes with pathogenic GLA mutations identified by screening renal, cardiac and stroke clinics, 1995-2017. J Med Genet. 2018;55:261-268. 
34. Ross LF, Saal HM, David KL, Anderson RR, American Academy of Pediatrics, American College of Medical Genetics and Genomics. Technical report: ethical and policy issues in genetic testing and screening of children. Genet Med. 2013;15: 234-245.

35. Botkin JR, Belmont JW, Berg JS, et al. Points to consider: ethical, legal, and psychosocial implications of genetic testing in children and adolescents. Am J Hum Genet. 2015;97:6-21.

36. Newborn Screening Translational Research Network. Longitudinal Pediatric Data Resource (LPDR). https://www.nbstrn.org/research-tools/ longitudinal-pediatric-data-resource, January 12, 2018.
37. Hall PL, Marquardt G, McHugh DM, et al. Postanalytical tools improve performance of newborn screening by tandem mass spectrometry. Genet Med. 2014;16:889-895.

38. Marquardt G, Currier R, McHugh DM, et al. Enhanced interpretation of newborn screening results without analyte cutoff values. Genet Med. 2012;14:648-655.

39. Mayo Clinic. Collaborative Laboratory Interpretive Reports. https://clir. mayo.edu, July 12, 2017

40. Minter Baerg MM, Stoway SD, et al. Precision newborn screening for lysosomal disorders. Genet Med. 2017 November 9. [Epub ahead of print] https://doi.org/10.1038/gim.2017.194 